\title{
Editorial for the Special Issue "Advanced Artificial Intelligence and Deep Learning for Remote Sensing"
}

\author{
Gwanggil Jeon
}

check for

Citation: Jeon, G. Editorial for the Special Issue "Advanced Artificial Intelligence and Deep Learning for Remote Sensing". Remote Sens. 2021, 13, 2883. https://doi.org/10.3390/ rs13152883

Received: 14 July 2021

Accepted: 20 July 2021

Published: 23 July 2021

Publisher's Note: MDPI stays neutra with regard to jurisdictional claims in published maps and institutional affiliations.

Copyright: (C) 2021 by the author. Licensee MDPI, Basel, Switzerland. This article is an open access article distributed under the terms and conditions of the Creative Commons Attribution (CC BY) license (https:/ / creativecommons.org/licenses/by/ $4.0 /)$.
Department of Embedded Systems Engineering, College of Information Technology, Incheon National University, 119 Academy-ro, Yeonsu-gu, Incheon 22012, Korea; gjeon@inu.ac.kr

Remote sensing is a fundamental tool for comprehending the earth and supporting human-earth communications. Artificial intelligence applications and data science techniques lead new research chances in various fields, such as remote sensing, which uses next fifth-generation communications and IoT. In the remote sensing area, the system- and human-generated information bring a massive amount of data, while new levels of accuracy, complexity, security, achievement, and reliability are requested. To this end, applicable and consistent research on artificial intelligence and data mining-based methods are needed. These methods can be both general and specific tools of artificial intelligence, including regression models, neural networks, decision trees, information retrieval, reinforcement learning, graphical models, and decision processes. We trust artificial intelligence and data science methods will provide promising tools to many challenging issues in remote sensing in terms of accuracy and reliability. In light of both these and many other challenges, a Special Issue of "Advanced Artificial Intelligence and Deep Learning for Remote Sensing" has been dedicated in order to address the current status, challenges, and future research priorities for the remote sensing area.

Starting from the above considerations, this Special Issue aims to report the latest advances and trends concerning the advanced artificial learning and data science techniques for remote sensing data processing issues. This edition of the Special Issue is focused primarily on artificial intelligence and deep learning for remote sensing applications, with special emphasis on stream processing and imaging platforms. This issue is intended to provide a highly recognized international forum to present recent advances in remote sensing. We welcomed both theoretical contributions as well as papers describing interesting applications. Papers were invited for this Special Issue considering aspects of this problem, including:

- Artificial intelligence and a data science approach for remote sensing;

- Reinforcement learning for remote sensing;

- Information retrieval for remote sensing;

- Big data analytics for beyond 5G;

- Edge/fog computing for remote sensing;

- IoT data analytics in remote sensing;

- Data-driven applications in remote sensing.

After review, a total of seven papers have been accepted for publication in this issue.

The evaporation duct is a weather phenomenon that often occurs in marine environments and affects the operation of shipborne radar. The most important evaluation parameter is the evaporation duct height (EDH). Forecasting the EDH and adjusting the working parameters and modes of the radar system in advance can greatly improve the radar performance. Traditionally, short-term forecast methods have been used to estimate the EDH, which are characterized by a low time resolution and poor forecast accuracy. In the contribution by Han et al. [1] "Evaporation Duct Height Nowcasting in China's Yellow Sea Based on Deep Learning", a novel approach for EDH nowcasting is proposed based on the deep learning network and EDH data measured in the Yellow Sea, China. 
The factors that affect nowcasting were analyzed. The time resolution and forecast time were $5 \mathrm{~min}$ and $0-2 \mathrm{~h}$, respectively. The results show that their proposed method has a higher forecast accuracy than traditional time series forecasting methods and confirm its feasibility and effectiveness.

Vehicle density and technological development increase the need for road and pedestrian safety systems. Identifying problems and addressing them through the development of systems in order to reduce the number of accidents and loss of life is imperative. The contribution by Zadobrischi and Dimian [2] "Inter-Urban Analysis of Pedestrian and Drivers through a Vehicular Network Based on Hybrid Communications Embedded in a Portable Car System and Advanced Image Processing Technologies" proposes the analysis and management of dangerous situations with the help of systems and modules designed in this direction. The approach and classification of situations that can cause accidents is another feature analyzed in this paper, including detecting elements of a psychosomatic nature: analysis and detection of the conditions a driver goes through, pedestrian analysis, and maintaining a preventive approach, all of which are embedded in a modular architecture. The versatility and usefulness of such a system come through its ability to adapt to context and the ability to communicate with traffic safety systems, such as V2V (vehicle-to-vehicle), V2I (vehicle-to-infrastructure), V2X (vehicle-to-everything), and VLC (visible light communication). All these elements are found in the operation of the system and its ability to become a portable device dedicated to road safety based on (radio frequency) RF-VLC (visible light communication).

The oceanic tropospheric duct is a structure with an abnormal atmospheric refractive index. This structure severely affects the remote sensing detection capability of electromagnetic systems designed for an environment with normal atmospheric refraction. The propagation loss of electromagnetic waves in the oceanic duct is an important concept in oceanic duct research. Owing to the long-term stability and short-term irregular changes in marine environmental parameters, the propagation loss in oceanic ducts has nonstationary and multiscale time characteristics. In the contribution by Dang et al. [3] "Multiscale Decomposition Prediction of Propagation Loss in Oceanic Tropospheric Ducts", the authors propose a multiscale decomposition prediction method for predicting the propagation loss in oceanic tropospheric ducts. The prediction performance was verified by simulating propagation loss data with noise. Using different evaluation criteria, the experimental results indicated that the proposed method outperforms six other comparison methods. Under noisy conditions, ensemble empirical mode decomposition effectively disassembles the original propagation loss into a limited number of stable sequences with different scale characteristics. Accordingly, predictive modeling was conducted based on multiscale propagation loss characteristic sequences. Finally, the authors reconstructed the predicted result to obtain the predicted value of the propagation loss in the oceanic duct. Additionally, a genetic algorithm was used to improve the generalization ability of the proposed method while avoiding the nonlinear predictor from falling into a local optimum.

Deep convolutional neural networks (CNNs) are widely used in hyperspectral image (HSI) classification. However, the most successful CNN architectures are handcrafted, which need professional knowledge and consume a very significant amount of time. The contribution by Liu et al. [4] "Continuous Particle Swarm Optimization-Based Deep Learning Architecture Search for Hyperspectral Image Classification" was proposed to automatically design cell-based CNN architectures for HSI classification. In this work, the authors propose an efficient continuous evolutionary method, named CPSO-Net, which can dramatically accelerate optimal architecture generation by the optimization of weightsharing parameters. First, a SuperNet with all candidate operations is maintained to share the parameters for all individuals and is optimized by collecting the gradients of all individuals in the population. Second, a novel direct encoding strategy is devised to encode architectures into particles, which inherit the parameters from the SuperNet. Then, particle swarm optimization is used to search for the optimal deep architecture from the particle swarm. Furthermore, experiments with limited training samples based on four widely used 
biased and unbiased hyperspectral datasets showed that their proposed method achieves a good performance that is comparable to the state-of-the-art HSI classification methods.

Deep learning methods have been shown to significantly improve the performance of building extraction from optical remote sensing imagery. However, keeping the morphological characteristics, especially the boundaries, is still a challenge that requires further study. In the contribution by He and Jiang [5] "Boundary-Assisted Learning for Building Extraction from Optical Remote Sensing Imagery", the authors propose a novel fully convolutional network (FCN) for accurately extracting buildings, in which a boundary learning task is embedded to help maintain the boundaries of buildings. Specifically, in the training phase, their framework simultaneously learns the extraction of buildings and boundary detection and only outputs extraction results while testing. In addition, the authors introduce spatial variation fusion (SVF) to establish an association between the two tasks, thus coupling them and making them both share the latent semantics and interact with each other. On the other hand, the authors utilize a separable convolution with a larger kernel to enlarge the receptive fields while reducing the number of model parameters and to adopt the convolutional block attention module (CBAM) to boost the network. The proposed framework was extensively evaluated on the WHU Building Dataset and the Inria Aerial Image Labeling Dataset. The experiments demonstrate that their method achieves a state-of-the-art performance on building extraction. With the assistance of boundary learning, the boundary maintenance of buildings is ameliorated.

Combined geometric positioning using images with different resolutions and imaging sensors is being increasingly widely utilized in practical engineering applications. In the contribution by Song et al. [6] "Combined Geometric Positioning and Performance Analysis of Multi-Resolution Optical Imageries from Satellite and Aerial Platforms Based on Weighted RFM Bundle Adjustment", the authors attempt to perform a combined geometric positioning and performance analysis of multi-resolution optical images from satellite and aerial platforms based on a weighted rational function model (RFM) bundle adjustment without using ground control points (GCPs). Firstly, the authors introduced an integrated image matching method combining least squares and phase correlation. Next, for the bundle adjustment, a combined model of the geometric positioning based on the weighted RFM bundle adjustment was derived, and a method for weight determination was given in order to make the weights of all image points variable. Finally, the authors conducted experiments using a case study in Shanghai with ZiYuan-3 (ZY-3) satellite imagery, GeoEye1 satellite imagery, and Digital Mapping Camera (DMC) aerial imagery to validate the effectiveness of the proposed weighted method, and to investigate the positioning accuracy by using different combination scenarios of multi-resolution heterogeneous images. The experimental results indicate that the proposed weighted method is effective, and that the positioning accuracy of different combination scenarios can give a good reference for the combined geometric positioning of multi-stereo heterogeneous images in future practical engineering applications.

In recent years, deep neural network (DNN)-based scene classification methods have achieved promising performances. However, the data-driven training strategy requires a large number of labeled samples, making the DNN-based methods unable to solve the scene classification problem in the case of a small number of labeled images. As the number and variety of scene images continue to grow, the cost and difficulty of manual annotation also increase. Therefore, it is significant to deal with the scene classification problem with only a few labeled samples. In the contribution by Li et al. [7] "AMN: Attention Metric Network for One-Shot Remote Sensing Image Scene Classification", the authors propose an attention metric network (AMN) in the framework of the few-shot learning (FSL) to improve the performance of one-shot scene classification. AMN is composed of a selfattention embedding network (SAEN) and a cross-attention metric network (CAMN). In SAEN, the authors adopt the spatial attention and the channel attention of feature maps in order to obtain abundant features of scene images. In CAMN, the authors propose a novel cross-attention mechanism which can highlight the features that are more concerned about 
different categories and improve the similarity measurement performance. A loss function combining mean square error loss with multi-class N-pair loss is developed, which helps to promote the intra-class similarity and inter-class variance of embedding features, and also improve the similarity measurement results. Experiments on the NWPU-RESISC45 dataset and the RSD-WHU46 dataset demonstrate that their method achieves state-of-the-art results on one-shot remote sensing image scene classification tasks.

The articles presented in this Special Issue provide insights in fields related to "Advanced Artificial Intelligence and Deep Learning for Remote Sensing", including models, performance evaluation and improvements, and application developments. We wish the readers can benefit from the insights of these papers, and contribute to these rapidly growing areas. We also hope that this Special Issue sheds light on major developments in the area of remote sensing and attracts attention by the scientific community in order to pursue further investigations leading to the rapid implementation of these technologies.

Funding: This research received no external funding.

Acknowledgments: We would like to express our appreciation to all the authors for their informative contributions and the reviewers.

Conflicts of Interest: The authors declare no conflict of interest.

\section{References}

1. Han, J.; Wu, J.-J.; Zhu, Q.-L.; Wang, H.-G.; Zhou, Y.-F.; Jiang, M.-B.; Zhang, S.-B.; Wang, B. Evaporation Duct Height Nowcasting in China's Yellow Sea Based on Deep Learning. Remote Sens. 2021, 13, 1577. [CrossRef]

2. Zadobrischi, E.; Dimian, M. Inter-Urban Analysis of Pedestrian and Drivers through a Vehicular Network Based on Hybrid Communications Embedded in a Portable Car System and Advanced Image Processing Technologies. Remote Sens. 2021, 13, 1234. [CrossRef]

3. Dang, M.; Wu, J.; Cui, S.; Guo, X.; Cao, Y.; Wei, H.; Wu, Z. Multiscale Decomposition Prediction of Propagation Loss in Oceanic Tropospheric Ducts. Remote Sens. 2021,13, 1173. [CrossRef]

4. Liu, X.; Zhang, C.; Cai, Z.; Yang, J.; Zhou, Z.; Gong, X. Continuous Particle Swarm Optimization-Based Deep Learning Architecture Search for Hyperspectral Image Classification. Remote Sens. 2021, 13, 1082. [CrossRef]

5. He, S.; Jiang, W. Boundary-Assisted Learning for Building Extraction from Optical Remote Sensing Imagery. Remote Sens. 2021, 13, 760. [CrossRef]

6. Song, W.; Liu, S.; Tong, X.; Niu, C.; Ye, Z.; Jin, Y. Combined Geometric Positioning and Performance Analysis of Multi-Resolution Optical Imageries from Satellite and Aerial Platforms Based on Weighted RFM Bundle Adjustment. Remote Sens. 2021, 13, 620. [CrossRef]

7. Li, X.; Pu, F.; Yang, R.; Gui, R.; Xu, X. AMN: Attention Metric Network for One-Shot Remote Sensing Image Scene Classification. Remote Sens. 2020, 12, 4046. [CrossRef] 